\title{
Driver-friendly motion control based on electric power steering and in-wheel motors on electric vehicle
}

\author{
R. Minaki* \\ Department of Electrical Engineering \\ and Information Systems (EEIS), \\ Graduate School of Engineering, \\ The University of Tokyo, \\ Rm. 7H1, P.O. Box 702, Hori Laboratory, \\ Transdisciplinary Sciences Bldg., \\ 5-1-5 Kashiwanoha, Kashiwa, Chiba, 277-8561, Japan \\ E-mail: minaki@hori.k.u-tokyo.ac.jp \\ ${ }^{*}$ Corresponding author

\section{Y. Hori} \\ Department of Advanced Energy, \\ Graduate School of Frontier Sciences, \\ Division of Transdisciplinary Sciences, \\ The University of Tokyo, \\ Rm. 7E1, P.O. Box 305, \\ Transdisciplinary Sciences Bldg., \\ 5-1-5 Kashiwanoha, Kashiwa, Chiba, 277-8561, Japan \\ E-mail: hori@k.u-tokyo.ac.jp
}

\begin{abstract}
This paper proposes to control driver's steering sensitivity and vehicle yaw motion based on human sensorial characteristics. Firstly, linearisation control of steering sensitivity enables the driver to recognize vehicle and road state via reaction torque of a steering wheel. It is power assist based on human kinesthetic sense by electric power steering. Secondly, linearisation control of vehicle yaw motion with in-wheel motors. Humans feel rotational motion by semicircular canal and vision. A driver controls yaw motion with the steering wheel. Therefore, this control increases operational performance for the vehicle. These controls will provide human-friendly manmachine interface to the driver.
\end{abstract}

Keywords: EPS; electric power steering, electric vehicle; human-friendly; IWM; In-Wheel-Motor, man-machine interface; steering control; vehicle motion control; human sensitivity, reaction torque; yaw rate.

Reference to this paper should be made as follows: Minaki, R. and Hori, Y. (2013) 'Driver-friendly motion control based on electric power steering and inwheel motors on electric vehicle', Int. J. Vehicle Autonomous Systems, Vol. 11, Nos. 2/3, pp.245-260. 
Biographical notes: Ryo Minaki received his Bachelor's Degree in electronic and computer engineering from the Tokyo Denki University in 2003. He worked as an electric circuit hardware design engineer in a subsidiary corporation of NEC during 2003-2007. He received his Master's Degree in electronic and computer engineering from the Tokyo Denki University in 2009. Since 2009, he has been a PhD student at the University of Tokyo, Tokyo, Japan.

Yoichi Hori received his $\mathrm{BS}, \mathrm{MS}$ and $\mathrm{PhD}$ Degrees in electrical engineering from The University of Tokyo, Tokyo, Japan, in 1978, 1980 and 1983, respectively. In 1983, he joined the Department of Electrical Engineering, the University of Tokyo, as a Research Associate, and later became an Assistant Professor, an Associate Professor, and, in 2000, a Professor. In 2008, he moved to the Department of Advanced Energy, Graduate School of Frontier Sciences, the University of Tokyo. He has been an IEEE Fellow since 2005 and also a fellow of the Institute of Electrical Engineering of Japan since 2011. $\mathrm{He}$ is currently the Director on Technological Developments of the Society of Automotive Engineers of Japan, the President of the Capacitors Forum, and the Chairman of the Motor Technology Symposium of the Japan Management Association.

\section{Introduction}

In today's world, the effectiveness of electronic active control systems in stabilising a vehicle's motion has been recognised; thus, numerous active control systems have been developed. Mass produced vehicles are equipped with Antilock Brake System (ABS). The Direct Yaw Control (DYC) has been developed to stabilise vehicle dynamics. However, according to traffic accident reports prepared by the Institute for Traffic Accident Research and Data Analysis (ITARDA) in Japan, almost all traffic accidents are caused by human error, such as driver negligence or operational mistakes. Therefore, future technologies need to be able to precisely detect driver's mistakes and adopt active control to increase vehicle safety. We are thinking of two methods to resolve the problem. The first one is constructing a traffic system without a driver, because people certainly make mistakes. It is the Intelligent Transport System (ITS) incorporating infrastructures to secure safety. The vehicles have an automatic driving-system-by-radar cruise to reduce driver's mistakes. That is to say, it is a position closer to railway systems. The other idea is assist drivers by informing them of operation mistakes and helping them to avoid dangerous situations. This paper is based on the latter thinking. We propose a driver-friendly motion control by using Electric Power Steering (EPS) and differential torque with In-Wheel-Motors (IWMs) on electric vehicles. This system actively feeds back the vehicle's state and road information as reaction torque to the driver. In addition, it controls vehicle motion characteristics dynamically with EPS and the differential torque. And it proposes an Active Front Steering (AFS) control based on power assist, without variable gear ratio steering and by-wire techniques. We have verified the effectiveness of this system. 


\section{Vehicle dynamics equations}

The driver controls a vehicle's dynamics by operating the front wheel angle with a steering wheel. A two-dimensional vehicle model is shown in Figure 1. Vehicle parameters in Figure 1 are shown in Table.1. The following equations can be calculated as two wheel model when right-and-left tyres are same characteristic. In this paper, the electric vehicle is equipped with two IWMs in the rear tyres. It has two input variables: the front tyre angle $\theta_{t}$ and the differential torque $M$ with IWMs. When the vehicle is in rotational motion, the tyres sideslip. Then, lateral forces $F_{f}$ and $F_{r}$ in proportion to the sideslip angles $\alpha_{f}$ and $\alpha_{r}$, are generated between the tyres and the road. $F_{f}$ is the front lateral force, and $F_{r}$ is the rear one. The proportionality coefficients are known as cornering stiffnesses $K_{f}$ and $K_{r}$. The lateral force equations are shown in Figures (1) and (2). The rear wheel steering is not operated. The lateral forces $F_{f}$ and $F_{r}$ generate yaw moment $\gamma$ around the centre of gravity of the vehicle. The lateral motion equation is shown in Figure (3), and the rotational motion equation is shown in Figure (4). $I_{z}$ is the body yaw moment of inertia. $l_{f}$ and $l_{r}$ are the distances between the centre of gravity and the front tyre or the rear one.

Figure 1 Vehicle model

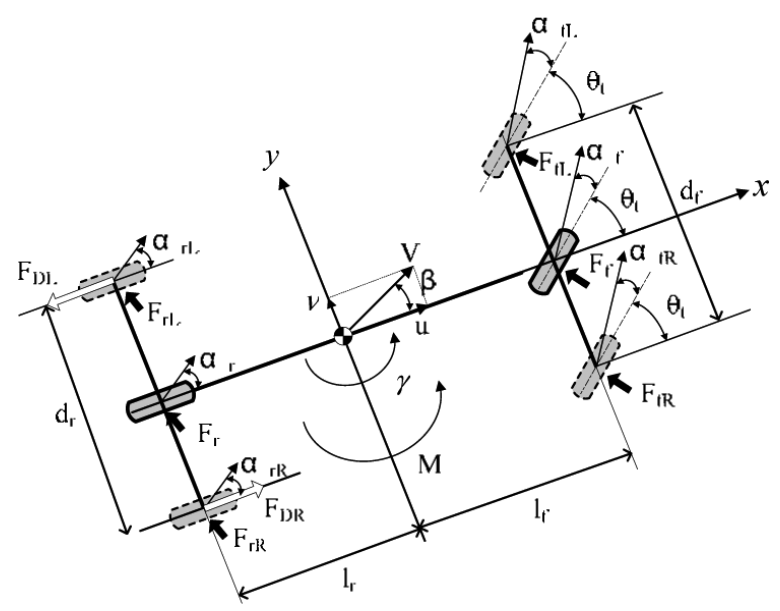

Figure 2(a) Driving force observer (see online version for colours)

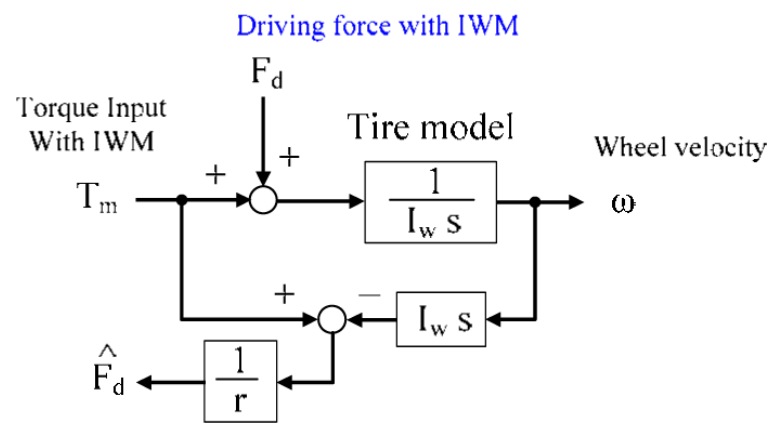


Figure 2(b) Steering system

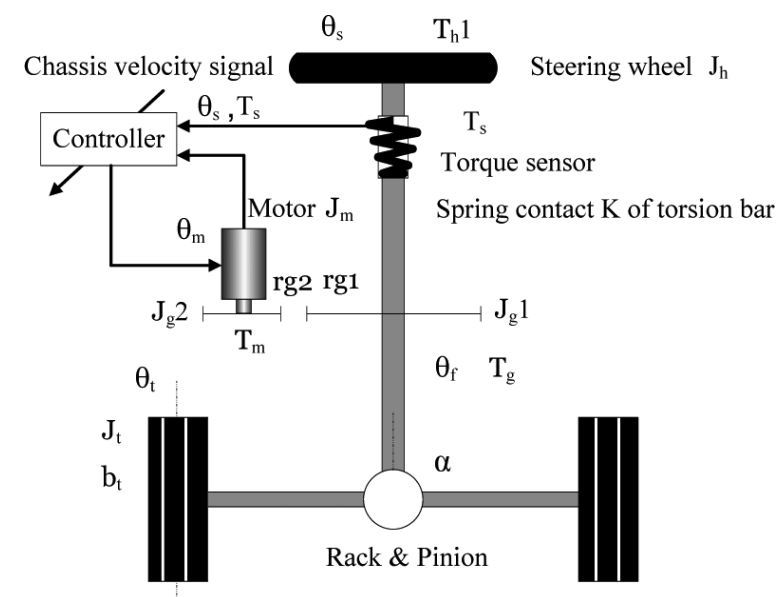

Figure 3 Linear block diagram of EPS system (see online version for colours)

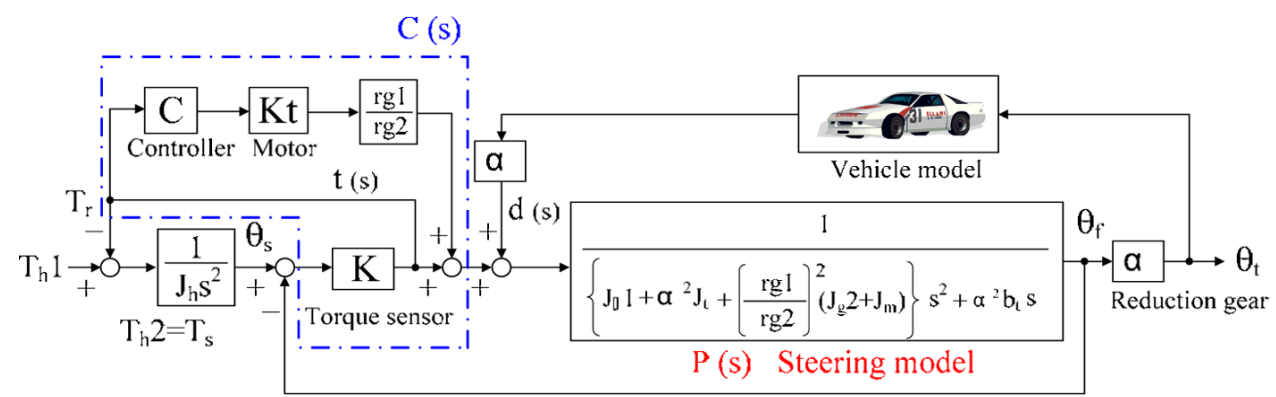

Figure 4 Simplified block diagram of EPS system

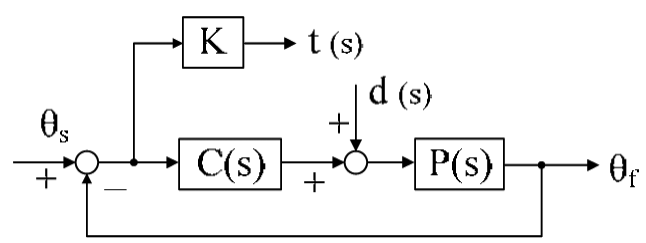

Table 1 Vehicle system parameters

\begin{tabular}{lc}
\hline Body mass & $M_{z}$ \\
Body yaw moment of inertia & $I_{z}$ \\
Distance between front and rear axle & $l_{f}, l_{r}$ \\
Tread & $d_{f}, d_{r}$ \\
Cornering stiffness & $K_{f}, K_{r}$ \\
Vehicle speed & $V$ \\
Front wheel angle & $\theta_{t}$ \\
\hline
\end{tabular}


Table 1 Vehicle system parameters (continued)

\begin{tabular}{lc}
\hline Tyre slip angle & $\alpha_{f}, \alpha_{r}$ \\
Chassis slip angle & $B$ \\
Yaw rate & $\gamma$ \\
\hline$F_{f}=F_{f L}=F_{f R}=-K_{f}\left(\frac{V \beta+\ell_{f} \gamma}{V}-\theta_{t}\right)$ & $F_{r}=F_{r L}=F_{r R}=-K_{r}\left(\frac{V \beta-\ell_{r} \gamma}{V}\right)$ \\
$M_{z} V\left(\frac{\mathrm{d} \beta}{\mathrm{d} t}+\gamma\right)=\left(F_{f L}+F_{f R}\right)+\left(F_{r L}+F_{r R}\right)=2 F_{f}-2 F_{r}$ \\
$I_{z} \frac{\mathrm{d} \gamma}{\mathrm{d} t}=\ell_{f}\left(F_{f L}+F_{f R}\right)-\ell_{r}\left(F_{r L}+F_{r R}\right)+M=2 \ell_{f} F_{f}-2 \ell_{r} F_{r}+M$.
\end{tabular}

$M$ is yaw moment by differential torque. In this study, the torque is directly generated with two IWMs in the rear wheels. The yaw moment equation is shown in Figure (5). $d_{r}$ is the rear wheelbase. $F_{D R}$ is the driving force of the right IWM, while $F_{D L}$ is the left one. The driving force $F_{D}$ shown in equation (6) is calculated by the motor torque $T_{m}$, wheel angular velocity $\dot{\omega}$, radius of a wheel $r$ and wheel inertia $I_{w}$ with the driving force observer shown in Figure 2. Motor torque is measured from motor current because the torque is a linear characteristic of the current. Wheel angular velocity is measured by a rotary encoder. $\tau_{s}$ is the time constant for the low pass filter to reduce sensor noise. equation (7) shows motor torque distribution with IWMs. $F_{x}$ is a longitudinal torque calculated from the acceleration pedal signal. IWMs output the torque in halves. The state equation for vehicle dynamics can be expressed in equation (8).

$$
\begin{aligned}
& M=\frac{d_{r}}{2}\left(F_{D R}-F_{D L}\right) \\
& \hat{F}_{D}=\left(\frac{T_{m}-I_{w} \quad \dot{\omega}}{r}\right) \frac{1}{1+\tau_{s} s} \\
& \left.\dot{x}=A \quad \begin{array}{l}
F_{D R} \\
F_{D L}
\end{array}\right]=\left[\begin{array}{ll}
\frac{1}{2} & \frac{2}{d_{r}} \\
\frac{1}{2} & -\frac{2}{d_{r}}
\end{array}\right]\left[\begin{array}{l}
F_{x} \\
M
\end{array}\right] . \\
& A=\left[\begin{array}{cc}
B \theta_{t}+H M, & x=[\beta \\
-2 \frac{K_{f}+K_{r}}{M_{z} V} & -1-2 \frac{\ell_{f} K_{f}-\ell_{r} K_{r}}{M_{z} V^{2}} \\
-2 \frac{l_{f} K_{f}-l_{r} K_{r}}{I_{z}} & -2 \frac{\ell_{f}^{2} K_{f}+\ell_{r}^{2} K_{r}}{I_{z} V}
\end{array}\right]
\end{aligned}
$$




$$
B=\left[\begin{array}{cc}
\frac{2 K_{f}}{M_{z} V} & \frac{2 \ell_{f} K_{f}}{I_{z}}
\end{array}\right]^{t}, \quad H=\left[\begin{array}{ll}
0 & \frac{1}{I_{z}}
\end{array}\right]^{t} .
$$

Figure 5 Steering and disturbance sensitivity design based on 2DOF

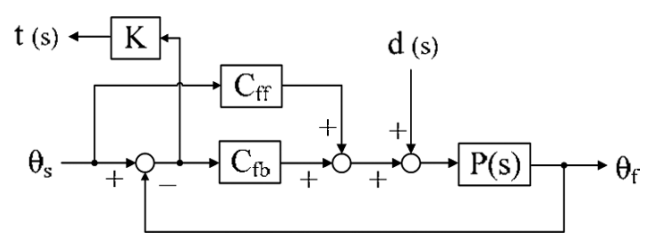

\section{Electric power steering}

An Electric Power Steering (EPS) system with electric motors not only reduces the load of the driver's operation, but also controls vehicle dynamics and reduces disturbance. In this chapter, the dynamic equation of EPS is introduced. The system diagram is shown in Figure 3 and the steering parameters are shown in Table 2. The driver turns the steering wheel and controls vehicle motion. Then, the EPS system calculates appropriate assist torque from the vehicle's velocity, the steering torque sensor and some vehicle motion sensors. It assists the driver's operation. After that, the front wheels are turned by the assist torque. At the same time, the front wheels receive the reaction torque from the road surface and the torque is transmitted to the driver. These are expressed in the following equations. First, the dynamics equations for gear 1 and 2 connected to the torsion bar spring and the assist motor are shown in Figures (9) and (10).

$$
\begin{aligned}
& (J g 2+J m) \frac{\mathrm{d}^{2} \theta_{2}}{\mathrm{~d} t^{2}}=T_{m}-\frac{r g 2}{2} F \\
& J g 1 \frac{\mathrm{d}^{2} \theta_{1}}{\mathrm{~d} t^{2}}=T_{s}-\frac{r g 1}{2} F-T_{g} .
\end{aligned}
$$

Table 2 Parameters of steering system

\begin{tabular}{lc}
\hline Steering wheel angle & $\theta_{s}$ \\
Motor angle & $\theta_{m}$ \\
Front axle wheel angle & $\theta_{f}$ \\
Front tyre angle & $\theta_{t}$ \\
Driver torque & $T_{h} 1$ \\
Steering torque & $T_{s}$ \\
Motor torque & $T_{m}$ \\
Rack torque & $T_{g}$ \\
Road reaction torque & $T_{d}$ \\
Steering wheel of inertia & $J_{h}$ \\
Motor of inertia & $J_{m}$ \\
\hline
\end{tabular}


Table 2 Parameters of steering system (continued)

\begin{tabular}{lc}
\hline Gear 1 of inertia & $J_{g} 1$ \\
Gear 2 of inertia & $J_{g} 2$ \\
Tyre of inertia & $J_{t}$ \\
Tyre friction & $B_{t}$ \\
Contact force between gear 1 and gear 2 & $F$ \\
Number of gear 1 teeth & $r g 1$ \\
Number of gear 2 teeth & $r g 2$ \\
Reduction gear ratio & $a$ \\
\hline
\end{tabular}

Equation (11) can be calculated from equations (9) and (10). In addition, equation (13) is calculated from equation (12), relating gear 1 to gear 2 in terms of the number of gear teeth.

$$
\begin{aligned}
& J g 1 \frac{\mathrm{d}^{2} \theta_{s}}{\mathrm{~d} t^{2}}=T_{s}+\left(\frac{r g 1}{r g 2}\right)\left\{(J g 2+J m) \frac{\mathrm{d}^{2} \theta_{m}}{\mathrm{~d} t^{2}}-T_{m}\right\}-T_{g} \\
& r g 1 \theta_{1}=-r g 2 \theta_{2} \\
& {\left[J g 1+\left(\frac{r g 1}{r g 2}\right)^{2}(J g 2+J m)\right] \frac{\mathrm{d}^{2} \theta_{s}}{\mathrm{~d} t^{2}}=T_{s}+\left(\frac{r g 1}{r g 2}\right) T_{m}-T_{g}}
\end{aligned}
$$

In this system, pinion gear inertia in the rack and pinion is ignored because the inertia is much smaller than the torque transmitted to the gear or the tyre inertia. For this reason, the rack torque can be expressed as in equation (14)

$$
T_{g}=\alpha^{2} J t \frac{\mathrm{d}^{2} \theta_{s}}{\mathrm{~d} t^{2}}+\alpha^{2} B_{t} \frac{\mathrm{d} \theta_{s}}{\mathrm{~d} t}-T_{d}
$$

Equation (15) can be calculated from equations (13) and (14)

$$
\left[J g 1+\alpha^{2} J t+\left(\frac{r g 1}{r g 2}\right)^{2}(J g 2+J m)\right] \frac{\mathrm{d}^{2} \theta_{s}}{\mathrm{~d} t^{2}}+\alpha^{2} B_{t} \frac{\mathrm{d} \theta_{s}}{\mathrm{~d} t}=T_{s}+\left(\frac{r g 1}{r g 2}\right) T_{m}+T_{d} .
$$

The EPS block diagram is shown in Figure 3. Plant $P(s)$ is the front steering model calculated in equation (15). The driver turns the front axle wheel angle and the vehicle motion via the steering wheel. The EPS system detects the steering torque by a torque sensor, such as a torsion bar spring. This sensor outputs torsional torque between the steering wheel angle and the front axle wheel angle. The torque is in proportion to the spring stiffness $K$. The electric motor outputs the power assist torque calculated with the EPS computer. And it controls the front axle wheel angle to follow the steering wheel angle. Then, the front wheel generates the lateral force proportion to a slip angle of the 
front wheel. At the same time, the front wheel receives the reaction torque as disturbance from the road surface. The driver receives the reaction torque via the steering wheel.

\section{Steering control based on two degrees of freedom and Variable Assist Torque}

In this chapter, we propose a steering control method based on driver sensitivity with EPS. This system feeds back vehicle state and road information as the reaction torque to the driver, and it helps the driver to avoid dangerous motions easily. Conventional techniques control the front wheel angle or yaw rate around the centre of gravity to follow the ideal yaw rate calculated in the vehicle dynamics equation. It is based on vehicle dynamics. Our proposed method is designed for the driver. The reaction torque has two components: necessary information for steering operation, and undesired disturbance torque to reject. We design a frequency-based two degree of freedom controller to divide those two components.

\subsection{Two degree of freedom control based on power assist and road disturbance sensitivity with EPS}

Figure 4 is a simplified EPS block diagram. In the figure, $R(s)$ is transfer function from the road disturbance torque to reaction torque transmitted to the driver. It is shown in equation (16).

$$
R(s)=\frac{t(s)}{d(s)}=K \frac{P(s)}{1+C(s) P(s)} .
$$

When divided by the torsion bar stiffness $K$, it agrees with the complementary sensitivity function $T(s)$ of the EPS system.

$$
T(s)=\frac{C(s) P(s)}{1+C(s) P(s)}
$$

This led to robust stability and disturbance sensitivity of the system.

The frequency of the reaction torque related to steering operation is less than $10 \mathrm{~Hz}$. On the other hand, the frequency of it to reduce appropriately is more than $10 \mathrm{~Hz}$. For this reason, the two degree of freedom control shown in Figure 5 is adopted to realise both the road disturbance sensitivity and the following capability to ideal vehicle dynamics. Feedback controller $C_{f b}$ compensates for the angle error between the steering wheel and the front axle wheel. The controller is designed to increase gain in frequencies higher than $10 \mathrm{~Hz}$ to reduce the disturbance torque (Figure 6). The equation is shown in equation (18).

$$
C_{f b}(s)=\frac{c s}{a s+b} .
$$


Figure 6 Bode diagram of control $C_{f b}$ (see online version for colours)

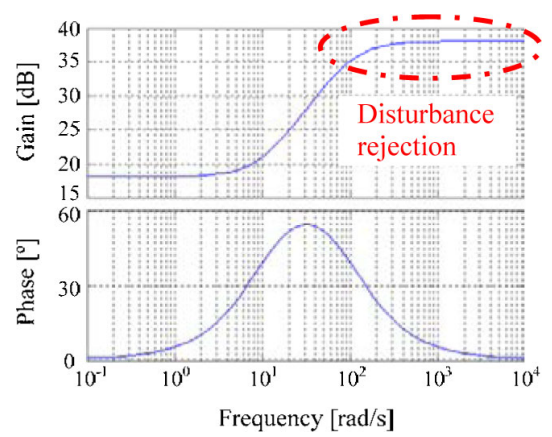

The driver operates the steering wheel while receiving the reaction torque and adjusting the steering torque. The feedfoward controller $C_{f f}$ compensates the reference value intended by the driver. The controller is designed to increase gain in the lower frequency than $10 \mathrm{~Hz}$ to assist driver torque. (Figure 7) The equation is shown in equation (19).

$$
C_{f f}(s)=\frac{c^{\prime}}{a^{\prime} s+b^{\prime}}
$$

Figure 7 Bode diagram of controller $C_{f f}$ (see online version for colours)

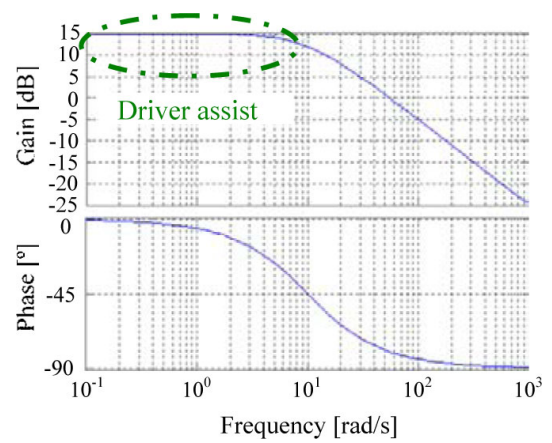

\subsection{Variable Assist Torque (VAT) control for linearising driver sensitivity and vehicle dynamics with EPS}

This paper proposes a Variable Assist Torque (VAT) control to linearise the driver's steering sensitivity. The control block diagram is shown in Figure $8 . C_{\mathrm{VAT}}$ is a controller of VAT to steering torque. We define steering sensitivity as in equation (20). It is the torque slope between the driver's torque input $T_{h} 1$ and the reaction torque $T_{r}$ which is transmitted to a steering wheel. It is linearised by the variable torque assist control shown in equations (23) and (24). The driver feels the slope with his steering operation. However, human sensitivity is a non-linear characteristic in general. It is approximated by equation (21). $T_{r e}$ is a torque which the driver feels. If a driver applied force to a steering wheel strongly, the driver may not feel the reaction torque compared to the driver's torque. We define driver sensitivity as in equation (22). This technique makes 
the assist torque variable to the steering torque $T_{s}$ and vehicle velocity $V$. And the graph is shown in Figure 9. Driver sensitivity linearisation enables the driver to recognise vehicle state and tyre conditions such as slip or grip phenomenon, because it relates to the principle of superposition. The equation includes the exponential function. Human sensitivity is a non-linear characteristic. When a human applies external torque $T_{h} 1$ to the steering wheel, the torque $T_{r e}$ which the human feels is a non-linear characteristic for the external torque. The equation relating $T_{h} 1$ to $T_{r e}$ is shown in equation (21). Natural logarithm is an inverse function for exponential function. For this reason, power assist torque using an exponential function can linearise the reaction torque transmitted to the drive to the driver's external torque. The linearity reduces the steering load to operate vehicle motion. In addition, the transfer function from the front wheel angle $\theta_{t}$ to yaw rate $\gamma$ is shown in equation (25). Human feels rotational motion by semicircular canal and vision. In case of a vehicle, a driver controls yaw motion with a steering wheel. This function is a first order system. When the yaw moment $M$ is zero, $l_{f}=l_{r}=1$, and $K_{f}=K_{r}=K_{y}$ this equation can be calculated from (5). The equation shows that yaw rate gain is determined from vehicle velocity $V$, and yaw rate response is determined from cornering stiffness $K_{y}$. In Figure 10, this lissajous curve compares the vehicle's yaw rates on dry roads with is yaw rates on wet roads. The abscissa axis is the steering wheel angle. And the vertical axis is a vehicle yaw rate. When the vehicle travels on wet roads, the hysteresis of the lissajous curve is wider than that dry roads, since cornering stiffness has been low on wet roads. From equation (25), the yaw rate has been delayed. As a result, the driver turns the steering wheel additionally, and the front tyre undergoes a slip phenomenon. Therefore, steering control needs driver sensitivity linearisation to feedback on the vehicle state and road information via the reaction torque, and assists a driver in avoiding dangerous states. The steering sensitivity factor $\alpha_{s}$ is determined from assist feeling for a driver.

$$
\begin{aligned}
& \text { Steering sensitivity } \frac{\mathrm{d} T_{r}}{\mathrm{~d} T_{h} 1} \\
& T_{r e}=\ln \left(T_{h} 1\right) \\
& \text { Driver sensitivity } \frac{d T_{r e}}{d T_{h} 1} \\
& T_{a}=\operatorname{sgn}\left(T_{s}\right) \cdot K_{V} \cdot\left(e^{\alpha_{s} T_{s}}-e^{\alpha_{s} T_{0}}\right) \\
& K_{v}=\frac{K_{a} \quad V_{\max }}{V_{\max }+V} \\
& P_{\gamma t}(s)=\frac{\gamma}{\theta_{t}}=\frac{1}{\frac{I_{z}}{K_{y} l} s+\frac{\ell}{V}} .
\end{aligned}
$$


Figure 8 Block diagram of variable assist torque control

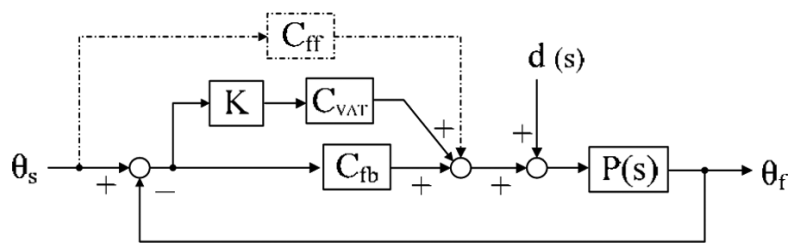

Figure 9 Variable power assist torque characteristic (see online version for colours)

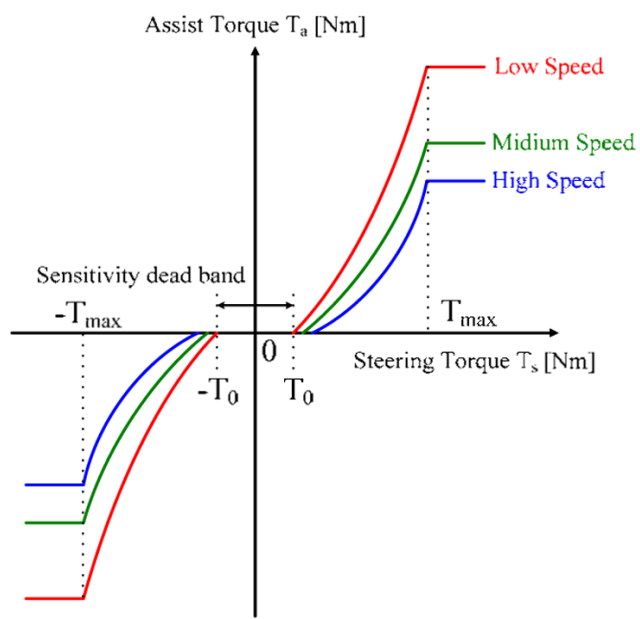

Figure 10 Steering angle vs. yaw rate characteristic (see online version for colours)

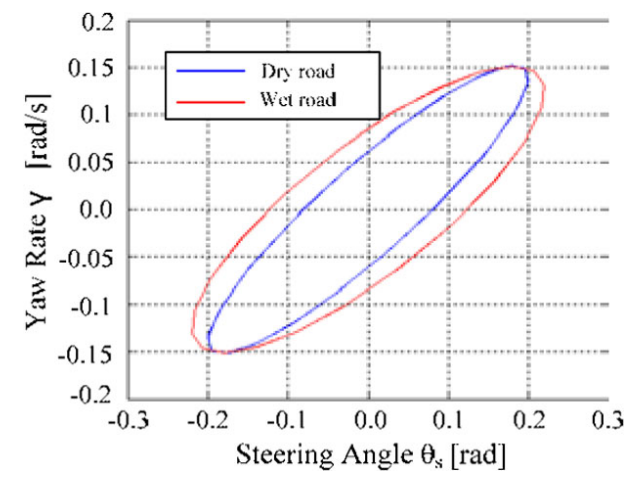

\section{Direct yaw moment control based on differential torque with IWMs}

The experimental electric vehicle COMS is equipped with two IWMs in the rear wheels. A differential force input with IWMs generates yaw moment $M$ around the centre of gravity on EV. The equation has been shown in equation (5). In addition, the transfer function $P_{\gamma M}$ from the yaw moment $M$ to the yaw rate $\gamma$ is shown in equation (26), when the front wheel angle input is zero, $l_{f}=l_{r}=1$, and $K_{f}=K_{r}=K_{y}$. It shows that yaw rate gain 
is determined by vehicle velocity $V$ and cornering stiffness $K_{y}$. If a driver operates a vehicle on a low friction road, the cornering stiffness would be small and the vehicle would slip. For this reason, when road friction is low, our proposed method uses yaw moments with IWMs to assist cornering motions with the steering system. The yaw moment to the steering wheel is shown in equation (27). It consists of yaw moment gain $G_{r}$ and distance between wheel and centre of gravity as shown in equation (28). It linearises the yaw rate to the steering wheel angle. And it enables the driver to operate the vehicle friendly. Direct yaw moment compensates yaw rate gain and time delay for road condition. The motor torque distribution of IWMs for the yaw moment $M$ is shown in equation (7).

$$
\begin{aligned}
& P_{\gamma M}=\frac{\gamma}{M}=\frac{1}{I_{z} s+\frac{K_{y} \ell^{2}}{V}} \\
& M=G_{\gamma} l \theta_{s} \\
& G_{\gamma}=\frac{K_{\gamma} V_{\max }}{V_{\max }+V} .
\end{aligned}
$$

\section{Simulation verification}

In this paper, we adopt the COMS produced by Toyota Auto Body as experimental vehicle. The COMS, shown in Figure 11, is a compact electric vehicle equipped with two IWMs operated independently in the right and left wheels. The parameter of the EV is shown in Table.3. The experiment simulates lane changes. The vehicle velocity is $40 \mathrm{~km} / \mathrm{h}$. The driver operates the steering wheel at sinusoidal input. The frequency of the input is $0.5 \mathrm{~Hz}$. The maximum motor torque with IWM is $150 \mathrm{Nm}$.

Table 3 Parameter of EV

\begin{tabular}{lcc}
\hline Body mass & $M_{z}$ & $350 \mathrm{~kg}$ \\
Body yaw moment of inertia & $I_{z}$ & $300 \mathrm{kgm}^{2}$ \\
Distance between front axle and centre of gravity & $l_{f}$ & $0.6 \mathrm{~m}$ \\
Distance between rear axle and centre of gravity & $l_{r}$ & $0.5 \mathrm{~m}$ \\
Front tread & $d_{f}$ & $0.7 \mathrm{~m}$ \\
Rear tread & $d_{r}$ & $0.6 \mathrm{~m}$ \\
Front cornering stiffness & $K_{f}$ & $10000 \mathrm{Nm} / \mathrm{rad}$ \\
Rear cornering stiffness & $K_{r}$ & $15000 \mathrm{Nm} / \mathrm{rad}$ \\
Maximum torque In-Wheel-Motor & $T_{\max }$ & $150 \mathrm{Nm}$ \\
Maximum vehicle velocity & $V_{\max }$ & $60 \mathrm{~km} / \mathrm{h}$ \\
\hline
\end{tabular}


Figure 11 Experimental electric vehicle: (a) EV COMS with EPS system and IWMs; (b) EPS system and (c) In-Wheels-Motors (see online version for colours)

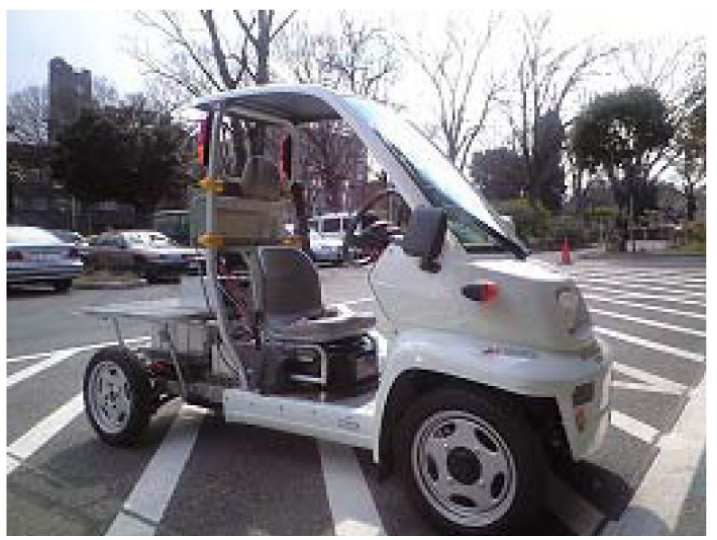

(a)

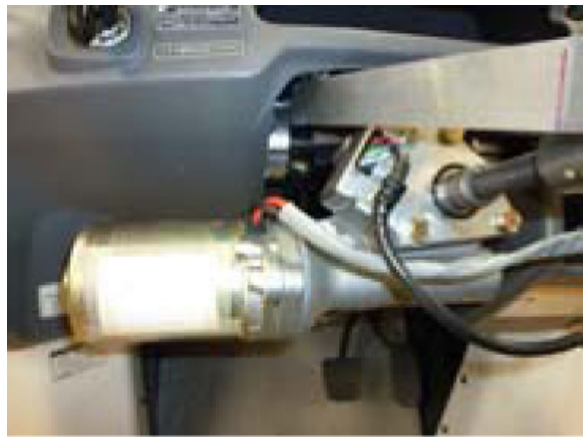

(b)

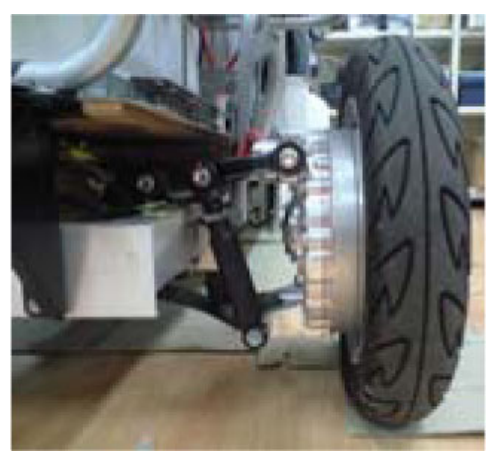

(c)

\subsection{Simulation verification of reaction torque control based on $2 D O F$ control for road disturbance sensitivity}

In this chapter, when mechanical disturbance torque to reduce is added from the front wheels, the reaction torque to the driver, the steering wheel angle and yaw rate in the vehicle are verified. The frequency of the disturbance is $15 \mathrm{~Hz}$. The result of conventional control without the disturbance sensitivity design is shown in Figure 12. This control could not reduce the disturbance sufficiently. And the disturbance vibration has been transmitted to the driver. As a result, the steering wheel angle and yaw rate have vibrated. Our proposed method, showed in Figure13, could reduce disturbance appropriately. When adding the required disturbance for steering operation at $5 \mathrm{~Hz}$, the disturbance has not been reduced and the driver receives the disturbance as road information. (Figure 14) The disturbance sensitivity design based on 2DOF control achieves division of the disturbance to reduce from the required disturbance for the driver. As a result, it enables the driver to feel a human-friendly interface with the vehicle. 
Figure 12 Simulation result of conventional EPS system in case of disturbance input at $15 \mathrm{~Hz}$ (see online version for colours)
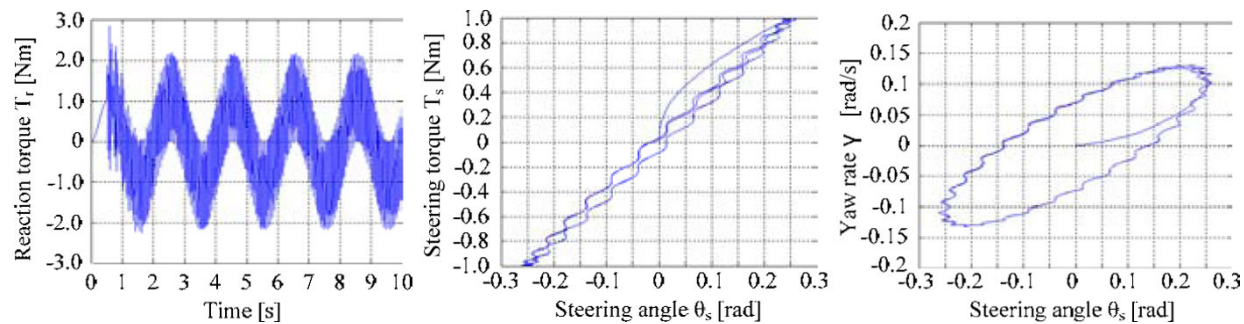

Figure 13 Simulation result of 2DOF control based on disturbance sensitivity in case of disturbance input at $15 \mathrm{~Hz}$ (see online version for colours)
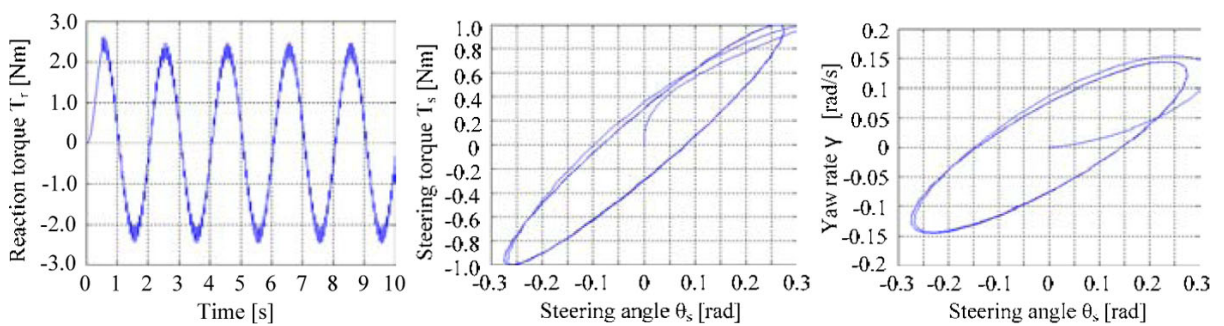

Figure 14 Simulation result of 2DOF control based on disturbance sensitivity in case of disturbance input $5 \mathrm{~Hz}$ (see online version for colours)
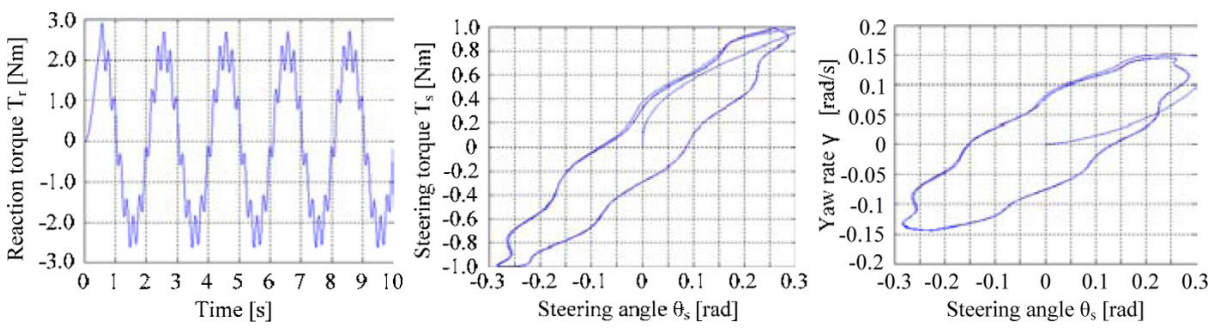

\subsection{Simulation verification of variable assist torque control based on linearisation of driver sensitivity}

The result of 2DOF VAT control (2DOF-VATC) is shown in Figure 15-(b). The result of only 2DOF control is shown in Figure 15-(a). Figure 15-(1) shows the driver sensitivity characteristic from driver input torque $T_{d}$ to reaction torque $T_{r e}$ which the driver feels. The characteristic of 2DOF-VATC is more linear than that of 2DOF control only. In case of 2DOF-VATC, non-linear power assist torque control is adopted to the steering torque to linearise the reaction torque for the driver. It enables the driver to recognise vehicle dynamics and road information because this information is contained in the reaction torque. When these lissajous figures are compared to the steering wheel angle and the steering torque in Figure 15-(2), the hysteresis characteristic of 2DOF-VATC is narrower than that of only 2DOF control. About the reverse steering operation at $\pm 0.6-0.8 \mathrm{rad}$ without VATC, the hysteresis band of the steering torque or the yaw rate is expanded. It makes the driver's reverse steering operation more difficult. In case of the 
2DOF-VATC, since the power assist torques become larger than the steering torque, the driver could reverse steer easily.

Figure 15 Experimental result of steering characteristics in case of steering input $0.5 \mathrm{~Hz}$ (see online version for colours) (1) Driver torque $T_{h} 1$ vs. Driver sensitivity torque $T_{r e}$ : (a) 2DOF control; (b) VAT control and (c) VAT control w/IWMs; (2) Steering angle $\theta_{s}$ vs. Steering torque $T_{s}$ : (a) 2DOF control; (b) VAT control and (c) VAT control w/IWMs and (3) Steering angle $\theta_{\mathrm{s}}$ vs. Yaw rate $\gamma$. (a) 2DOF control; (b) VAT control and (c) VAT control w/IWMs
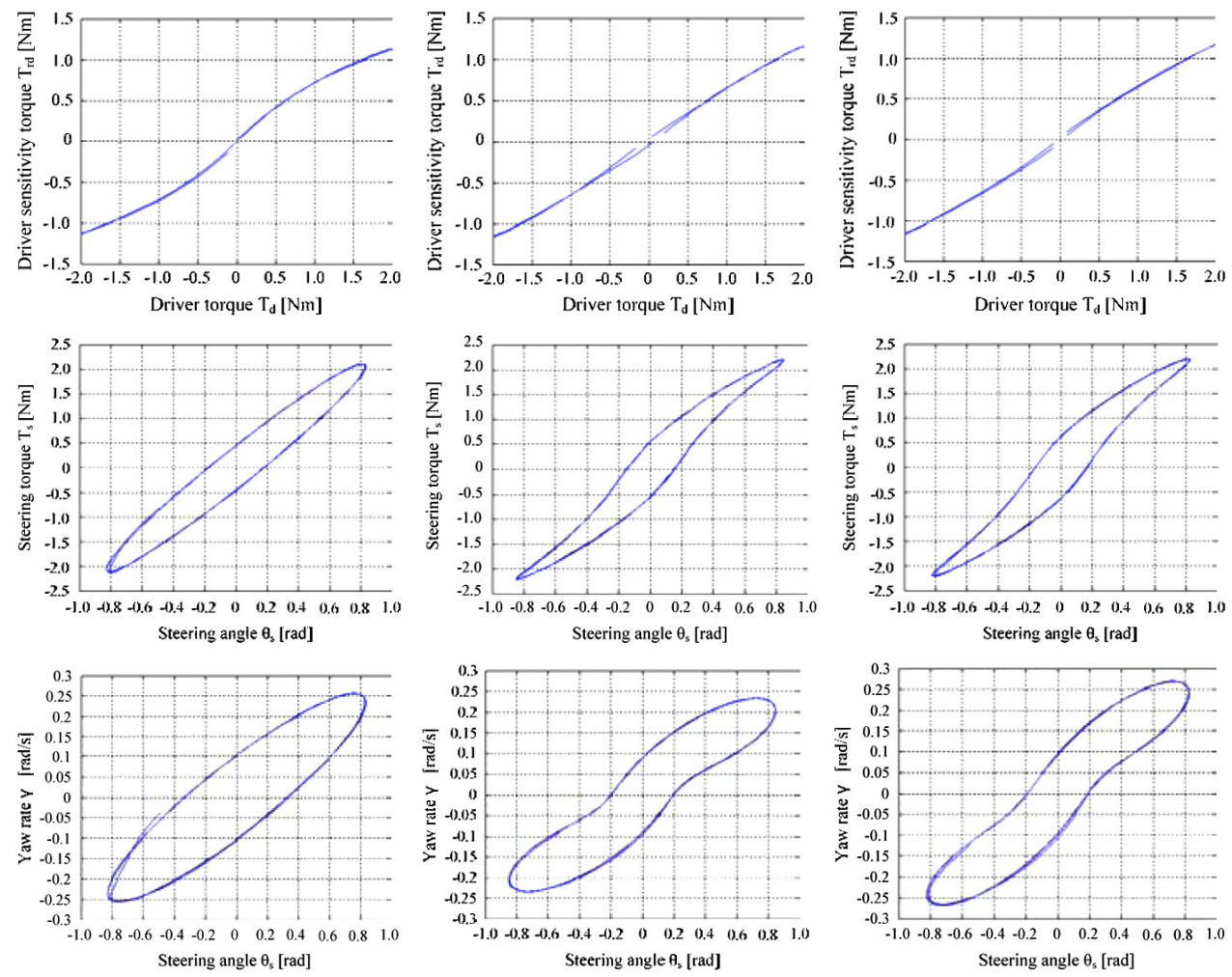

\subsection{Simulation verification of direct yaw rate control combined with electric power steering and In-Wheel-Motors on electric vehicle}

Electric power steering systems control 2DOF variable torque assist controls (2DOFVATC). In addition, the result added to the differential torque control with IWMs is shown in Figure 15-(c). The yaw moment is directly controlled by the differential torque. For this reason, it could increase the yaw rate gain without the steering operation in Figure 15-(3)-(c). And it almost maintains the hysteresis characteristic and linearity of driver sensitivity to the same extent as that of VATC in Figure 15-(1)-(c). In Figure 15-(2)-(c), the hysteresis band has been a little expanded. It shows that the driver receives reaction torque of yaw moment dynamics via the steering wheel. The driver feels that the steering wheel has been heavy and operates the vehicle carefully. This is an advantage for driver-friendly man-machine interface. Utilising both EPS and differential torque with 
IWMs, we achieve extension of the vehicle dynamics range, compared to conventional vehicles.

\section{Conclusion}

We propose a driver-friendly vehicle motion control based on an EPS system and IWM in an electric vehicle, to reduce driver operational mistakes and traffic accidents. First, a 2DOF control based on the disturbance sensitivity design divides the required disturbance for steering operation from the disturbance to reduce appropriately in frequency ranges with the EPS system. Second, the VAT control linearises driver sensitivity from reaction torque to driver input torque. These methods enable the driver to recognise vehicle dynamics and road information via reaction torque. They provides driver-friendly interfaces with the vehicle. Finally, a differential torque with IWMs directly linearises the yaw rate response to increase operational performance for the driver. Human-friendly interface combined with EPS and IWMs enables the driver to predict risk of the vehicle and road state via reaction torque and to avoid the risk.

\section{References}

Abe, M. (2008) Vehicle Dynamics and Control, Denki University Publication, Tokyo.

Ando, N. and Fujimoto, H. (2011) 'Yaw-rate control for electric vehicle with active front/rear steering and driving/braking force distribution of rear wheels', IEEJ Transactions on Industry Applications, Vol. 131, No. 4, pp.616-623.

Aoki, K. and Hori, Y. (2005) 'Reactive torque control in active steering with planetary gear', IEE of Japan Technical Meeting Record, VT-05-15, January, Tokyo, Japan.

Endo, S. and Kobayashi, H. (2004) 'EPS control technology', NSK Technical Journal Motion \& Control, No. 16, pp.51-56.

Falcone, P., Borrelli, F., Asgari, J., Tseng, H. and Hrovat, D. (2007) 'Predictive active steering control for autonomous vehicle systems', IEEE Trans. Control Syst. Technol., Vol. 15, No. 3, May, pp.566-580.

Koehn, P. and Eckrich, M. (2004) Active Steering the BMW Approach Towards Modern Steering Technology, SAE technical paper, 01-1105.

Minaki, R. and Hori, Y. (2009) 'Experimental verification of driver-friendly reactive torque control based on driver sensitivity to active front steering', 35th Annual Conference of the IEEE Industrial Electronics Society, November, Porto, Portugal, pp.3091-3096.

Minaki, R. Hoshino, H. and Hori, Y. (2009) 'Ergonomic verification of reactive torque control based on driver's sensitivity characteristics for active front steering', 5th International IEEE Vehicle Power and Propulsion Conference, September, Bari, Italy, pp.160-165.

Nakano, S., Nishizaki, K., Nishihara, O. and Kumamoto, H. (2002) 'Study of steering control with steer-by-wire - improvement of vehicle's response by D* control', JSAE Trans., Vol. 33, No. 3, pp.121-124. 